\title{
Synergism between the mTOR inhibitor rapamycin and FAK down-regulation in the treatment of acute lymphoblastic leukemia
}

Pei-Jie Shi ${ }^{1 \dagger}$, Lu-Hong Xu ${ }^{1 \dagger}$, Kang-Yu Lin ${ }^{2}$, Wen-jun Weng ${ }^{1}$ and Jian-Pei Fang ${ }^{1 *}$

\begin{abstract}
Background: Acute lymphoblastic leukemia (ALL) is an aggressive malignant disorder of lymphoid progenitor cells in both children and adults. Although improvements in contemporary therapy and development of new treatment strategies have led to dramatic increases in the cure rate in children with $A L L$, the relapse rate remains high and the prognosis of relapsed childhood ALL is poor. Molecularly targeted therapies have emerged as the leading treatments in cancer therapy. Multi-cytotoxic drug regimens have achieved success, yet many studies addressing targeted therapies have focused on only one single agent. In this study, we attempted to investigate whether the effect of the mammalian target of rapamycin (MTOR) inhibitor rapamycin is synergistic with the effect of focal adhesion kinase (FAK) down-regulation in the treatment of ALL.

Methods: The effect of rapamycin combined with FAK down-regulation on cell proliferation, the cell cycle, and apoptosis was investigated in the human precursor B acute lymphoblastic leukemia cells REH and on survival time and leukemia progression in a non-obese diabetic/severe combined immunodeficiency (NOD/SCID) mouse model.

Results: When combined with FAK down-regulation, rapamycin-induced suppression of cell proliferation, $G_{0} / G_{1}$ cell cycle arrest, and apoptosis were significantly enhanced. In addition, REH cell-injected NOD/SCID mice treated with rapamycin and a short-hairpin RNA (shRNA) to down-regulate FAK had significantly longer survival times and slower leukemia progression compared with mice injected with REH-empty vector cells and treated with rapamycin. Moreover, the B-cell CLL/lymphoma-2 (BCL-2) gene family was shown to be involved in the enhancement, by combined treatment, of REH cell apoptosis.

Conclusions: FAK down-regulation enhanced the in vitro and in vivo inhibitory effects of rapamycin on REH cell growth, indicating that the simultaneous targeting of mTOR- and FAK-related pathways might offer a novel and powerful strategy for treating ALL.
\end{abstract}

Keywords: Acute lymphoblastic leukemia, Focal adhesion kinase, Mammalian target of rapamycin inhibitor, Therapeutic efficacy, REH cells

\section{Background}

Acute lymphoblastic leukemia (ALL) is an aggressive malignant disorder of lymphoid progenitor cells in both children and adults, and it is caused by genetic lesions in blood-progenitor cells $[1,2]$. Although improvements in contemporary therapy and development of new treatment

\footnotetext{
* Correspondence: jpfang2005@163.com

${ }^{\dagger}$ Equal contributors

${ }^{1}$ Department of Pediatrics, Sun Yat-sen Memorial Hospital, Sun Yat-sen University, No. 107, West Yan Jiang Road, Guangzhou, Guangdong 510120, China

Full list of author information is available at the end of the article
}

strategies [3-5] have led to dramatic increases in the cure rate in children, the relapse rate remains high and the prognosis of relapsed childhood ALL is poor [6]. Hematopoietic stem cell transplantation (HSCT) seems to be curative for patients with ALL relapse and high-risk acute lymphoblastic leukemia [7]. However, post-HSCT relapse is still an obstacle to therapeutic improvement $[8,9]$. Use of an antineoplastic immunosuppressive agent after transplantation should facilitate treatment. However, although immunosuppressive therapy can 
significantly increase the survival time of transplant patients, it also promotes tumor growth [10].

Rapamycin, an inhibitor of mammalian target of rapamycin (mTOR), is a bacterial macrolide that was originally used as an antifungal agent $[11,12]$. The findings that rapamycin targets mTOR and is also antiproliferative led to its use as an anticancer agent [10, 13]. Evidence indicates that the phosphatidylinositol 3-kinase (PI3K), Akt, mTOR signaling pathway (PI3K/Akt/ mTOR) is dysregulated in hematologic malignancies and abnormally activated in childhood ALL. Most commonly, this abnormal activation is due to constitutive activation of Akt and provides a compelling rationale to target this pathway in ALL. Preclinical studies demonstrating significant activity against ALL has led to a number of clinical trials [14]. Combination therapeutic strategies of using rapamycin with focal adhesion kinase (FAK) down-regulation may address the problem of resistance to mTOR-targeted monotherapy and improve the treatment effect.

FAK is a $125-\mathrm{kDa}$ non-receptor tyrosine kinase that plays an important role in cell survival, proliferation, apoptosis, migration, and invasion $[15,16]$. FAK expression is higher in malignant cells than in the corresponding normal cells [17], and a high expression of FAK is associated with enhanced blast migration and poor prognosis in acute myeloid leukemia (AML) [18]. Furthermore, in our previous study, FAK down-regulation inhibited leukemogenesis in breakpoint cluster region/ Abelson leukemia virus (BCR/ABL)-transformed ALL cells and increased apoptosis and drug efficacy in pro-B ALL cells. In vivo FAK down-regulation has also been shown to impair cell migration and inhibit leukemia progression [19]. Interestingly, FAK is an upstream kinase of Akt [20], indicating that FAK down-regulation might suppress rapamycin-induced Akt activation [21, 22]. This possibility provided us with a rationale for combining rapamycin with FAK down-regulation therapy to treat ALL in patients who received HSCT.

In the present study, we found that FAK was activated in tumor cells of ALL patients. Either FAK downregulation or rapamycin caused growth inhibition of a pro-B ALL cell line, and growth was more profoundly inhibited by a combination of FAK down-regulation and rapamycin. The combination enhanced the treatment effect of rapamycin, prolonged median survival time, and slowed the progression of leukemia in nonobese diabetic/severe combined immunodeficiency (NOD/SCID) mice injected with REH (the human precursor B acute lymphoblastic leukemia cell line) cells. Collectively, our results suggest that the combination of rapamycin and FAK down-regulation may be a promising therapeutic strategy in ALL patients who received HSCT.

\section{Results \\ FAK was highly expressed in leukemia cells of patients with ALL}

We examined whether FAK was activated in leukemia cells from patients and showed by Western blot analysis (Fig. 1a(i)) and confirmed by quantitative realtime polymerase chain reaction (PCR) analysis (Fig. 1a(ii)) that FAK protein expression is higher in leukemia cells from patients $(n=10)$ than in lymphocytes from normal volunteers $(n=3)$. The differences were statistically significant $(p<0.05)$.

\section{Rapamycin activated AKT and FAK down-regulation inhibited AKT phosphorylation}

To examine whether FAK down-regulation was necessary for rapamycin-mediated effects, AKT and phospho-AKT levels were assayed to test our hypothesis. Western blot was used to analyze REH-empty vector cells or REH cells knocked down with FAK short-hairpin RNA (shRNA) and then treated with or without rapamycin $100 \mathrm{nM}$ for $10 \mathrm{~h}$. As shown in Fig. 1b, AKT was activated by rapamycin and phospho-AKT was inhibited by the combination of rapamycin and FAK down-regulation.

\section{FAK down-regulation enhanced the growth-inhibitory effects of rapamycin}

To study the effect of FAK down-regulation on rapamycin efficacy, FAK was down-regulated in REH cells by lentiviral-GFP-FAK-shRNA (demonstrated previously to block FAK expression specifically [23]). Western blot and quantitative real-time PCR analyses demonstrated that retrovirus-mediated RNAi successfully inhibited FAK protein expression and significantly inhibited mRNA expression $(p<0.05$; Fig. $2 \mathrm{a}(\mathrm{ii}))$ by up to $50 \%$ relative to the empty vector in REH cells.

Then, REH-empty vector and REH-FAK shRNA cells were plated in 96-well plates and cultured with rapamycin $(0-1000 \mathrm{nM})$ for 48 or $72 \mathrm{~h}$, respectively, after which cell numbers and viability were evaluated with the Cell Counting Kit-8 (CCK-8) assay. The combination of FAK down-regulation and rapamycin treatment induced enhanced growth inhibition of REH cells compared with rapamycin treatment alone. Rapamycin $(100 \mathrm{nM}, 48 \mathrm{~h})$ alone inhibited the growth of REH cells by $30.19 \pm 1.35 \%$, while the combination of FAK down-regulation and rapamycin inhibited growth by $50.68 \pm 0.84 \%$. When these cells were exposed to rapamycin at the same concentration, the REH-FAK shRNA cells exhibited greater inhibition than the REH-empty vector cells. The results are shown in Fig. 2b. 

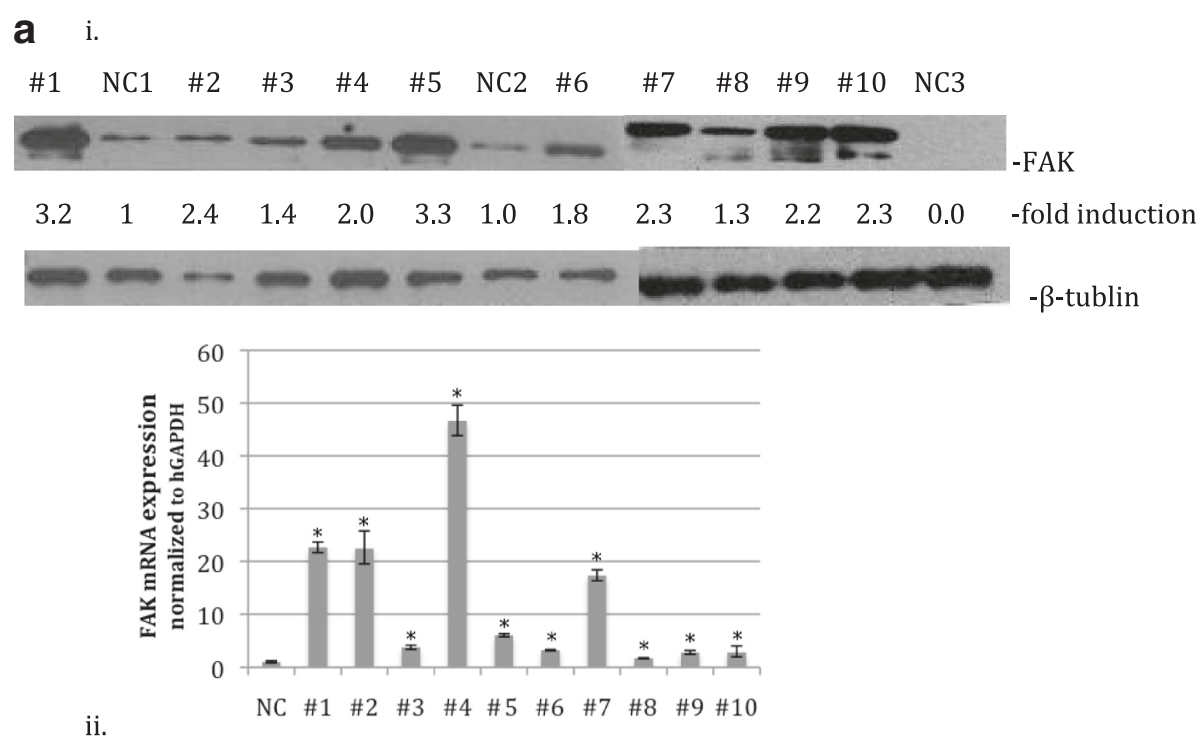

ii.

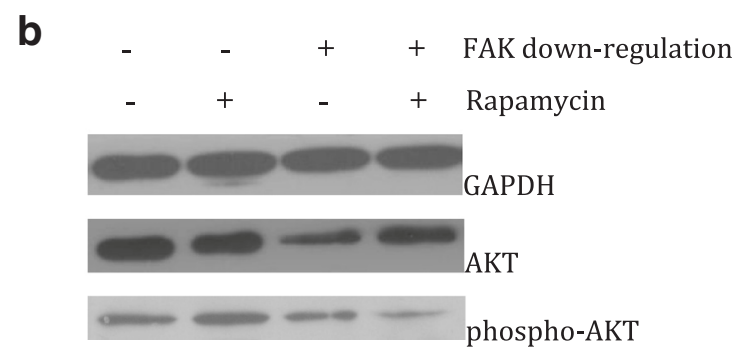

Fig. 1 a FAK is activated in the cells of ALL patients. i: Western blot analysis. Cell lysates from freshly isolated ALL (\#1-\#10) and normal control volunteer (NC1-NC3) cells were prepared and subjected to Western blot analysis. The polyvinylidene fluoride membrane was sequentially probed with anti-FAK. The band intensity was measured via densitometry. ii: Quantitative real-time PCR analysis also showed that the mRNA expression of FAK was increased in ALL patients compared with normal control volunteers. The differences were statistically significant $(p<0.05)$. b Rapamycin activated AKT and FAK down-regulation inhibited AKT phosphorylation. After treatment with or without 100 nM rapamycin for $10 \mathrm{~h}$, REH-empty vector cells or REH-FAK shRNA cells were subjected to Western blot analysis. AKT was activated by rapamycin, and phospho-AKT was inhibited by the combination treatment with rapamycin and FAK down-regulation

FAK down-regulation increased rapamycin-induced $G_{0} / G_{1}$ cell cycle arrest and cell apoptosis

To reveal the mechanism underlying REH cell growth inhibition by the combined treatment, the cell cycle and cell apoptosis were examined. Marked accumulation of REH cells in $G_{0} / G_{1}$ phase occurred, with a concomitant decrease in the number of cells in the $S$ phase after culture with rapamycin (100 nM, $48 \mathrm{~h})$ or FAK downregulation. Combined treatment further increased the percentage of cells in the $G_{0} / G_{1}$ phase and decreased that in the $\mathrm{S}$ phase $(p<0.05)$ (Fig. 3a). As shown in Fig. 3a, the percentages of cells in the $G_{0} / G_{1}$ cell cycle arrest in the rapamycin-only group and the combination group were $65.34 \pm 3.62 \%$ and $79.35 \pm 3.28 \%$, respectively. The differences were statistically significant $(p<0.05)$. We next determined the effects of rapamycin and FAK down-regulation on cell apoptosis. REH-empty vector cells or REH-FAK shRNA cells were treated with or without $100 \mathrm{nM}$ rapamycin for $30 \mathrm{~h}$, stained with annexin $\mathrm{V} /$ propidium iodide $(\mathrm{PI})$, and then analyzed by a flow cytometer. As shown in Fig. 3b, the percentage of annexin $\mathrm{V}^{+}$cells was significantly higher after the combination treatment than rapamycin treatment only $(17.50 \pm 0.55 \%$ vs $9.05 \pm 0.41 \%$, respectively; $p<0.05)$, showing that the combined treatment was a more effective driver of cells into the $\mathrm{G}_{0} / \mathrm{G}_{1}$ cell cycle arrest and cell apoptosis.

\section{Effect of rapamycin, FAK down-regulation, and their} combination on BCL-2 family mRNA expression in REH cells Subsequent experiments focused on the expression of apoptosis-related genes. B-cell CLL/lymphoma-2 (BCL2) family mRNA expression was examined in REHempty vector cells or REH-FAK shRNA cells treated 
a i. REH-empty vector REH-FAK shRNA
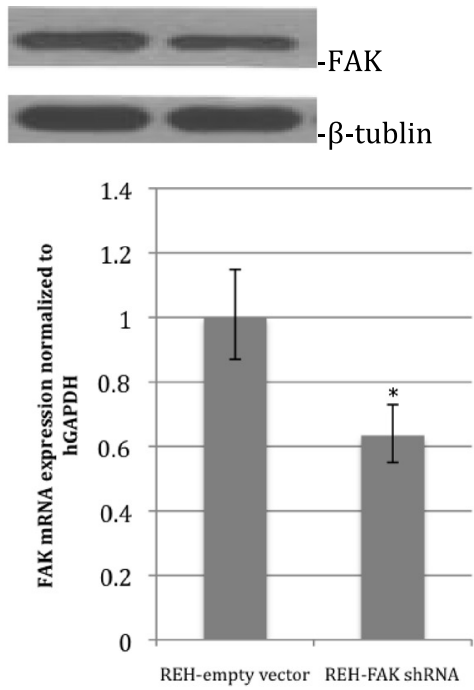

ii.
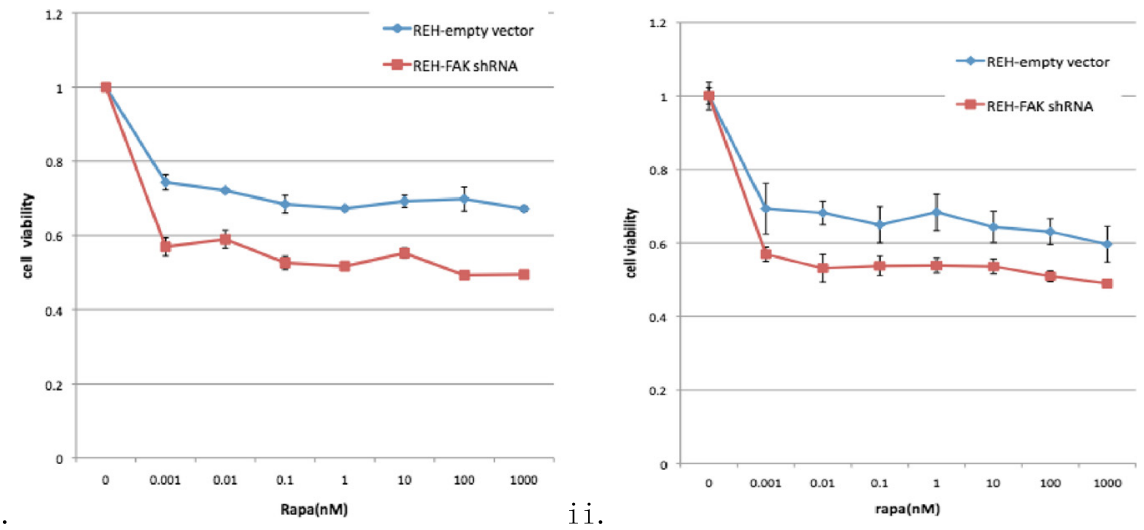

Fig. 2 Targeting FAK via RNAi enhanced the growth-inhibitory effects of rapamycin. a FAK protein (i) and mRNA expression (ii) in REH-empty vector and REH-FAK shRNA cells. b The mTOR inhibitor rapamycin and FAK down-regulation inhibited the proliferation of REH cells. REH-empty vector or REH-FAK shRNA cells were plated in 96-well plates and cultured with rapamycin (0-1000 nM). After 2 days (i) or 3 days (ii), the cells were treated with CCK-8 $(10 \mu \mathrm{l} /$ well) for $2 \mathrm{~h}$ and the absorbance was measured. The results represent the mean \pm S.D. of three experiments performed in triplicate

with or without rapamycin $100 \mathrm{nM}$ for $10 \mathrm{~h}$ using quantitative real-time PCR. As shown in Fig. 4, the expression of BCL-2 interacting killer (BIK) and other proapoptosis genes except BCL-2 antagonist killer (BAK) was significantly promoted by each treatment alone and significantly more by the combination therapy. Combined treatment resulted in a significantly higher mRNA expression of p53 up-regulated modulator of apoptosis (PUMA), BCL-2-modifying factor (BMF), and BCL-2associated $\mathrm{X}$ protein $(\mathrm{BAX})(p<0.05)$, a lower mRNA expression of the anti-apoptosis gene BCL-2, and a 17.55-fold higher BCL-2/BAX ratio. However, expression of the pro-apoptosis fragment myeloid cell leukemia-1 (MCL-1)S was only slightly increased by the combination treatment relative to rapamycin alone.

\section{FAK down-regulation enhanced the in vivo efficacy of} rapamycin

To further investigate the effects of FAK downregulation on rapamycin efficacy in vivo, NOD/SCID mice were intravenously injected with REH cells (REHempty vector cells or REH-FAK shRNA cells) and treated 10 days later with rapamycin $0.15 \mathrm{mg} / \mathrm{kg}$ for 7 days. All mice injected with REH cells died (Fig. 5a). With rapamycin treatment, death occurred between day 29 and day 52 with a median of 43 days $(n=11)$ in the REH-empty vector group and between day 43 and day 71 with a median of 57 days $(n=11)$ in the REH-FAK shRNA group. Without rapamycin treatment, the corresponding median survival times were 25 and 36 days, respectively $(n=3)$. Log-rank analysis showed 


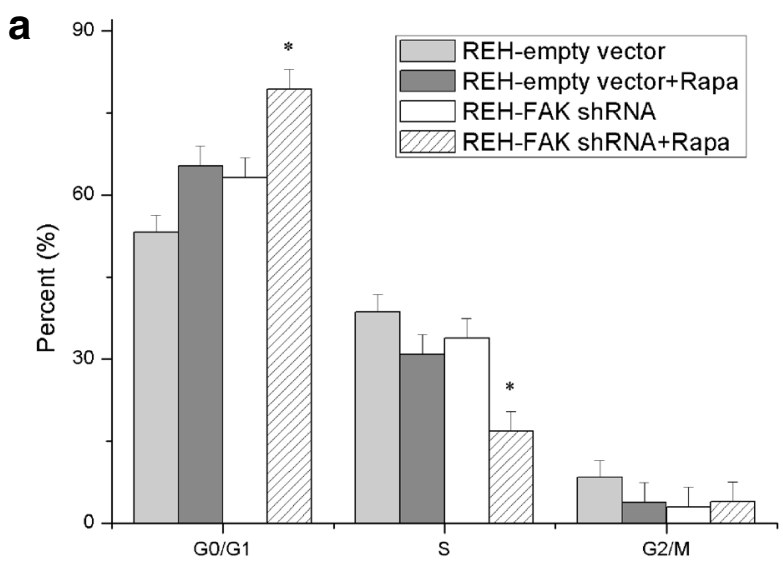

b

REH-empty vector

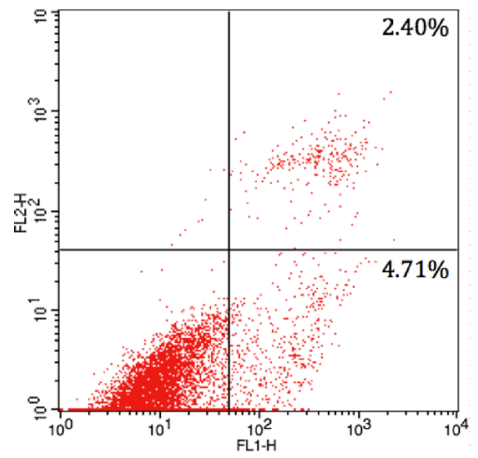

REH-FAK ShRNA

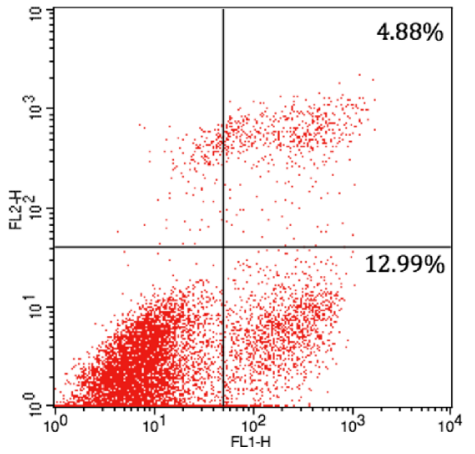

REH-empty vector + Rapa

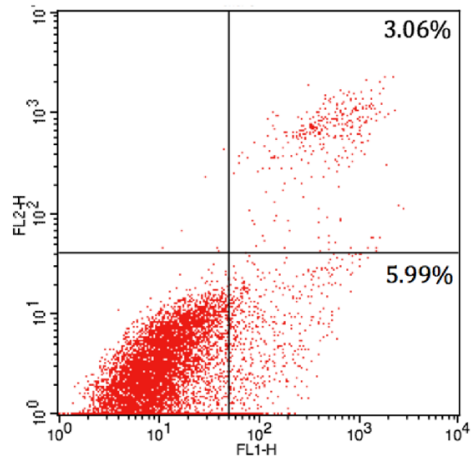

REH-FAK shRNA +Rapa

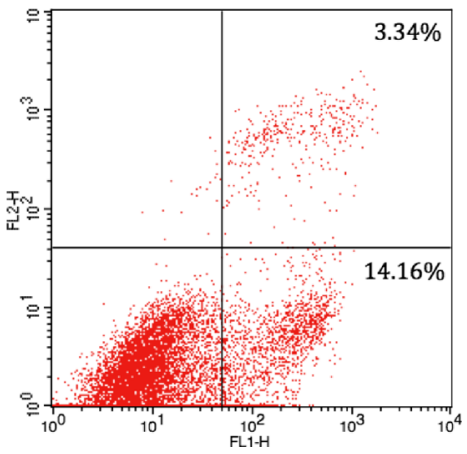

Fig. 3 a The combination of rapamycin and FAK down-regulation induced enhanced $G_{0} / G_{1}$ cell cycle arrest in REH cells. REH-empty vector or REH-FAK shRNA cells were cultured with or without rapamycin $(100 \mathrm{nM})$. After $48 \mathrm{~h}$, the cell cycle was analyzed in these cells. The statistical significance of differences between populations in the $G_{0} / G_{1}$ and $S$ phases of the cell cycle induced by either rapamycin or $F A K$ down-regulation alone or by combined treatment with both was determined through one-way analysis of variance (ANOVA) followed by Bonferroni's multiple comparison tests. The results represent the mean \pm S.D. of three experiments performed in triplicate. ${ }^{*} p<0.05$; Rapa, rapamycin. $\mathbf{b}$ The induction of apoptosis in REH cells by rapamycin and FAK down-regulation. REH-empty vector or REH-FAK shRNA cells were cultured with or without rapamycin $(100 \mathrm{nM})$. After $30 \mathrm{~h}$, cell apoptosis was analyzed using the annexin V/PI apoptosis detection kit. The combination of rapamycin and FAK down-regulation induced more cell apoptosis in REH cells than in rapamycin treatment alone

a significant difference in median survival time between the treated empty vector and FAK shRNA groups $(p<0.05)$, indicating that FAK down-regulation prolonged the survival of rapamycin-treated NOD/SCID mice.
White blood cells (WBCs counted on day 0 , day 10 , day 17 , day 24, and day 31 after REH cell injection) increased over time. Comparing the rapamycin-treated FAK shRNA group with the rapamycin-treated REH- 


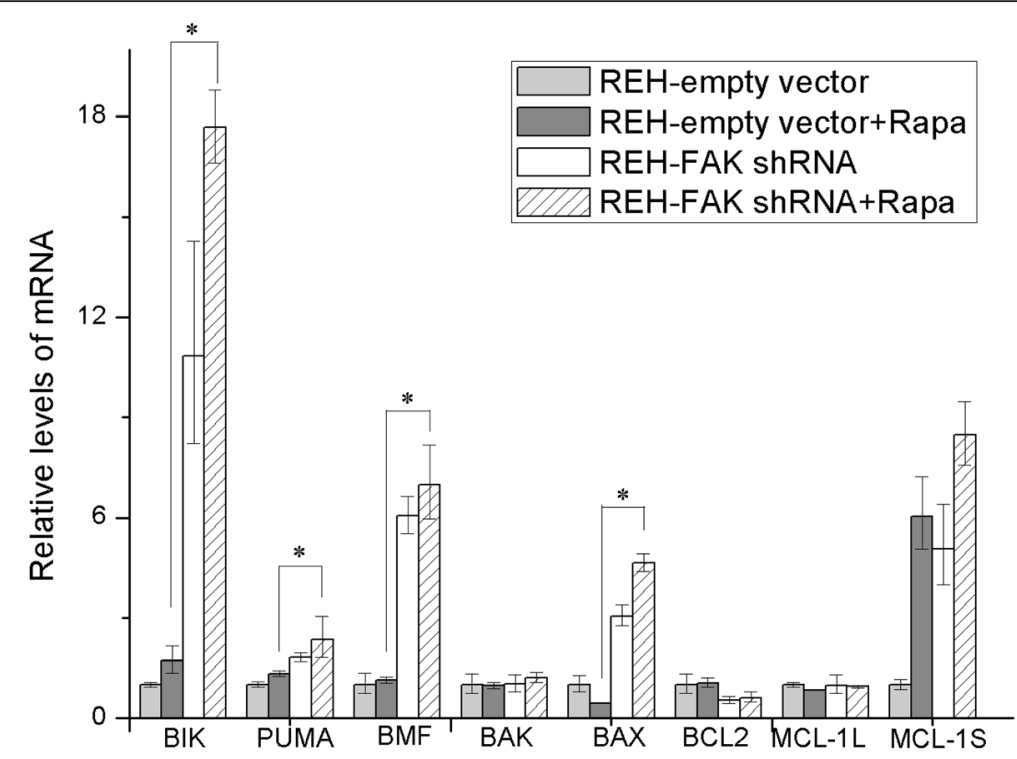

Fig. 4 Changes in BCL-2 family expression caused by rapamycin treatment and FAK down-regulation. REH-empty vector or REH-FAK shRNA cells were cultured with or without rapamycin (100 nM). After $10 \mathrm{~h}$, RNA was extracted from these cells and mRNA expression was detected using quantitative real-time PCR. The expression of pro-apoptosis genes, such as BIK, PUMA, BMF, BAX, and MCL-1S, was higher in the combination treatment group than in the rapamycin-only group. The expression of anti-apoptosis genes, such as BCL-2, was decreased in the combination treatment group compared with the rapamycin-only group. The results represent the mean \pm S.D. of three experiments performed in triplicate. ${ }^{*} p<0.05$; Rapa rapamycin

empty vector group, the rise in WBC count was significantly slower $(p<0.01$; Fig. $5 \mathrm{~b})$ and spleens removed from mice sacrificed on day 25 were much smaller in the rapamycin-treated FAK shRNA group (Fig. 5c), indicating that FAK down-regulation increases rapamycininduced inhibition of leukemia progression in vivo.

It is intriguing that none of the 22 mice treated with rapamycin developed neurological disorders such as paralysis pressing one side of the head close to the floor or vertigo, indicating rapamycin treatment might prevent central nervous system leukemia. All the neurological disorders described above were present and severe in the three mice injected with the REH-empty vector without rapamycin treatment and were present and less severe in only one mouse injected with REH-FAK shRNA without rapamycin treatment.

\section{Discussion}

In cancer therapy, combinatorial strategies are commonly used to treat malignancies that are resistant to standard treatment. Several combinations of rapamycin or rapalogs with other antitumor drugs have previously been investigated [24-26]. Recently, it was found that the cytotoxic drug carboplatin could enhance the effect of the mTOR inhibitor everolimus, and this combination is already undergoing phase I evaluation [27], which suggests that the clinical benefit from mTOR inhibitors could be maximized by combining them with other agents. In the present study, patients with ALL when compared to normal volunteers had varying degrees of FAK activation (Fig. 1a). In other studies, FAK was elevated in human AML, where increased FAK expression and activity were correlated with poor prognosis [18]. In our previous study, FAK down-regulation enhanced the drug efficacy of imatinib in ALL cells [19]. Herein, we assessed whether FAK could augment rapamycin efficacy in ALL cells, and the results were encouraging. In vitro, rapamycin combined with FAK down-regulation enhanced cell growth inhibition, $G_{0} / G_{1}$ cell cycle arrest, and cell apoptosis. In vivo, this combination slowed leukemia progression, indicating that FAK downregulation can improve the efficacy of rapamycin in ALL treatment.

We supposed that rapamycin blocked the mTOR pathway, attenuating p70S6K activation and leading to Akt pathway activation. The antitumor activity of rapamycin is compromised by the hyperactivity of the feedback-looprelevant PI3K/Akt signaling pathway [24]. Thus, when FAK is down-regulated, rapamycin-induced Akt phosphorylation is inhibited, which might explain how FAK down-regulation enhances the efficacy of rapamycin. The results in Fig. 1b strongly support this hypothesis.

The present finding that the blockade of mTOR by rapamycin and down-regulation of FAK inhibit proliferation of REH cells and increase $G_{0} / G_{1}$ cell cycle arrest (Figs. $2 \mathrm{~b}$ and $3 \mathrm{a}$ ) are consistent with the findings of 

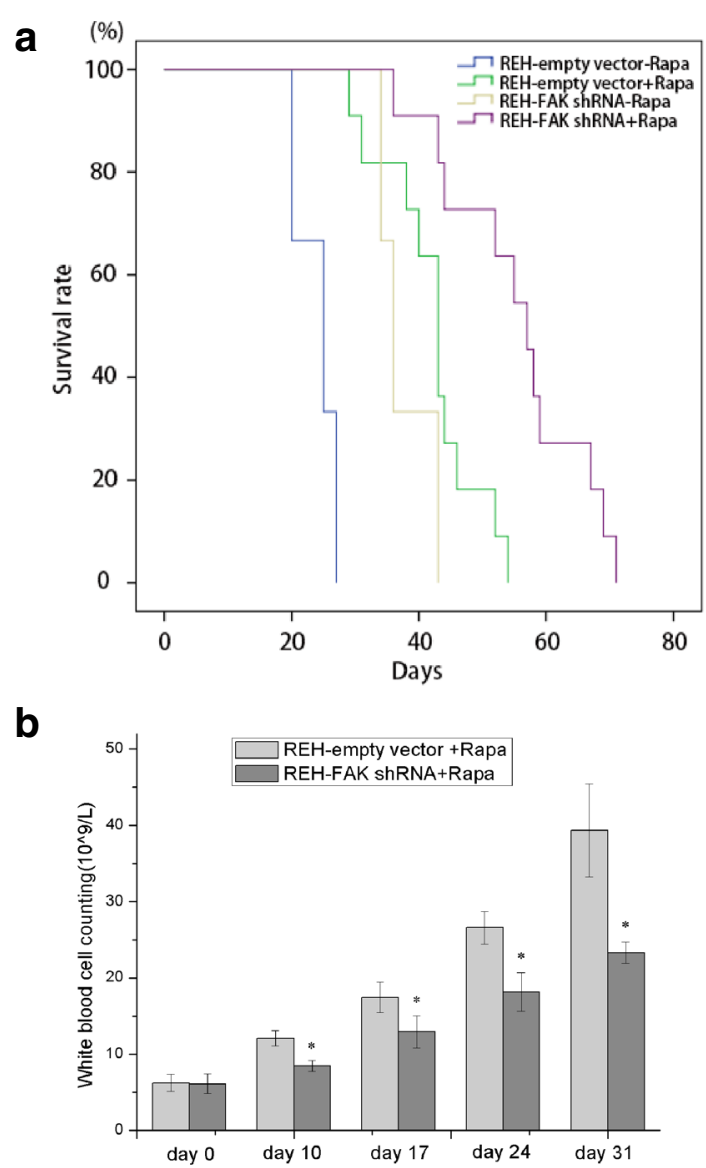

C

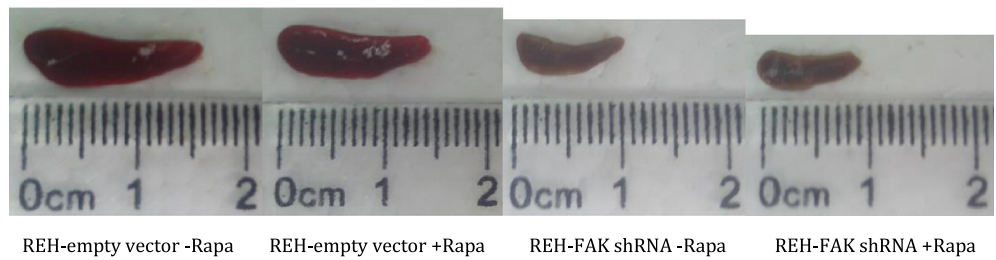

Fig. 5 FAK down-regulation enhanced rapamycin efficacy in vivo. Five million REH-empty vector or REH-FAK shRNA cells were injected into NOD/ SCID mice on day 0 . The mice were treated daily with $1.5 \mathrm{mg} / \mathrm{kg}$ rapamycin or DMSO for 7 days beginning on day 10 . The survivors were monitored daily, and leukemia progression was assayed on days 10, 17, 24, and 31. a Kaplan-Meier survival analysis showed that the combination of rapamycin and FAK down-regulation prolonged survival compared with rapamycin treatment alone. $\mathbf{b}$ The white blood cells in the peripheral blood were counted on days $0,10,17,24$, and 31 . The results showed that the combination of rapamycin and FAK down-regulation reduced the leukemia burden compared with rapamycin treatment alone. ${ }^{*} p<0.05$, the combination group vs. the rapamycin-only group. $\mathbf{c}$ Leukemic mice developed splenomegaly on day 25, but the combination of rapamycin and FAK down-regulation reduced the size of the spleens compared with rapamycin treatment alone

other groups. Recher et al. showed that rapamycin could inhibit the proliferation of acute myeloid leukemia cells and sensitize these cells to growth inhibition mediated by cytotoxic agents such as cytarabine [28]. In another study, LY294002 was shown to enhance rapamycinmediated inhibition of T-cell proliferation [29]. On the other hand, Pan et al. found that Caco- 2 cell proliferation was significantly decreased by inhibition of FAK gene expression [30]. These results support our observations.
In our analyses, the induction of ALL cell apoptosis by rapamycin was mild. However, when rapamycin was combined with FAK down-regulation, the cell apoptosis effect was dramatically enhanced (Fig. 3b), indicating that the enhancement by FAK down-regulation of rapamycin's antitumor efficacy is mainly due to apoptosis induction. Rapamycin treatment and/or FAK downregulation can stimulate the expression of the proapoptosis genes, such as BIK, PUMA, BMF, BAX, and 
MCL-1S, and inhibit the expression of the anti-apoptosis genes such as BCL-2 and BCL-XL (Fig. 4). We suppose that using the new BCL-2 inhibitors in combination therapy will boost treatment efficacy [31].

Using a murine model of leukemia induced by REH cells, we further investigated the effects of FAK downregulation on rapamycin efficacy in vivo. All the mice died after injection of leukemic cells despite treatment with rapamycin for 7 days. But compared with rapamycin treatment alone, rapamycin combined with FAK down-regulation prolonged median survival by 14 days, reduced spleen size, and diminished peripheral leukocyte count in the model mice. These findings suggest that FAK down-regulation potentiates rapamycin-induced inhibition of leukemia progression in vivo.

\section{Conclusions}

The present study reported a new ALL therapy involving FAK down-regulation combined with the mTOR inhibitor rapamycin. FAK down-regulation was found to potentiate rapamycin-induced suppression of ALL cell growth both in vitro and in vivo, suggesting a new concept of ALL treatment (i.e., the targeting of both mTORand FAK-related pathways) to achieve more powerful therapeutic effect. Since the lentivirus-vector delivery system is not practical for clinical use, we suggest the testing of FAK inhibitors in future experiments [32, 33]. Because the precise mechanism is still unclear, the interaction between mTOR and FAK pathways should be explored in the future.

\section{Methods}

\section{Cell culture and clinical samples}

The REH human acute lymphoblastic pro-B cell leukemia cell line (CRL8286, ATCC, USA) was cultured in RPMI1640 medium (Gibco, USA) supplemented with $10 \%$ fetal bovine serum (Gibco, USA) in a $\mathrm{CO}_{2}$ incubator. A total of 13 clinical samples, including samples from 8 patients with a primary diagnosis of acute lymphoblastic leukemia (ALL), 2 patients with relapsed ALL, and 3 normal samples from Sun Yat-sen Memorial Hospital, were included in this study. All cells were freshly isolated from the bone marrow of each individual. The clinical characteristics of these individuals are presented in Table 1. All patients provided informed consent, and the study was approved by the ethics committees of Sun Yat-sen Memorial Hospital.

Down-regulation of FAK with shRNA and establishment of stable transfected clones.

A short-hairpin RNA (shRNA)-expressing lentivirusvector delivery system was applied as previously described [34, 35]. The obtained lentiviruses, containing the GFP-FAK shRNA vector or a GFP-empty vector construct, were used for the transfection of REH cells. To
Table 1 Patient clinical characteristics

\begin{tabular}{llll}
\hline Patient & Age (years) & Sex & Disease status \\
\hline$\# 1$ & 12 & Male & ALL newly diagnosed \\
$\# 2$ & 3 & Female & ALL newly diagnosed \\
$\# 3$ & 2 & Male & ALL newly diagnosed \\
$\# 4$ & 7 & Female & ALL newly diagnosed \\
$\# 5$ & 9 & Female & ALL newly diagnosed \\
$\# 6$ & 7 & Female & ALL newly diagnosed \\
\#7 & 7 & Female & ALL newly diagnosed \\
\#8 & 8 & Male & ALL newly diagnosed \\
\#9 & 15 & Male & ALL relapse \\
\#10 & 13 & Female & ALL relapse \\
NC1 & 14 & Female & Normal control \\
NC2 & 15 & Male & Normal control \\
NC3 & 7 & Male & Normal control \\
\hline AL & 7 & N &
\end{tabular}

ALL acute lymphoblastic leukemia, NC normal control

establish stable transfected clones, the REH cells were sorted repeatedly based on a green fluorescent protein (GFP) expression using a flow cytometer (FACSAria, Becton Dickinson, CA) at $72 \mathrm{~h}$ after transfection, until the percentage of GFP-positive clones was greater than $99 \%$. The stably transfected clones were used for further experiments. Quantitative real-time PCR analysis revealed that the best silencing efficiency was achieved with the shRNA designated FAK X40-2 shRNA, and the FAK target sequence was 5 -GGAATGCTTCAAGTGTGCTT-3 .

\section{Reagents}

Rapamycin, a mammalian target of rapamycin (mTOR) inhibitor, was purchased from Sigma (USA). Rapamycin was dissolved in $100 \%$ dimethyl sulfoxide (DMSO) (Sigma, USA) to a stock concentration of $25 \mathrm{mg} / \mathrm{ml}$ and stored at $-20{ }^{\circ} \mathrm{C}$.

\section{Western blotting and quantitative real-time PCR}

The cells were lysed in radio immuno-precipitation assay (RIPA) buffer (Pierce, Rockford, IL, USA) with protease and phosphatase inhibitors (Roche, Beijing, China), and the supernatant was collected after centrifugation. Denatured proteins were fractionated via electrophoresis on a 10-12 \% sodium dodecyl sulfate (SDS) polyacrylamide gel and transferred to a methanol-activated polyvinylidene fluoride (PVDF) membrane (Millipore). The membrane was blocked for $2 \mathrm{~h}$ in Tris-buffered saline Tween-20 (TBST) containing $5 \%$ bovine serum albumin and then incubated with a polyclonal mouse anti-FAK (Millipore, USA), rabbit anti-AKT (Cell Signaling Technology, Boston, MA, USA), rabbit anti-phospho-AKT (Ser473, Cell Signaling Technology, Boston, MA, USA), rabbit anti-GAPDH (Cell Signaling Technology, Boston, 
MA, USA), or rabbit anti- $\beta$-tubulin (Cell Signaling Technology, Boston, MA, USA) antibody overnight at $4{ }^{\circ} \mathrm{C}$. One hour after incubation with the corresponding goat anti-mouse (Thermo) or goat anti-rabbit (Sigma) horseradish peroxidase-conjugated secondary antibody, the level of protein expression was detected using the enhanced chemiluminescence (ECL) method (Millipore, USA) according to the manufacturer's instructions.

Total RNA was extracted using the TRIzol reagent (Invitrogen, USA) according to the manufacturer's protocols. cDNA was prepared from $1 \mu \mathrm{g}$ of total RNA using a reverse transcription-polymerase chain reaction (RT-PCR) kit (Takara, Japan) with oligodT according to the manufacturer's instructions. cDNA samples were then analyzed via quantitative real-time PCR using SYBR Green (Takara, Japan) in an ABI Step One Real-Time PCR machine (Applied Biosystems, Foster City, CA), with 40 cycles of $95{ }^{\circ} \mathrm{C}$ for $15 \mathrm{~s}$ and $60{ }^{\circ} \mathrm{C}$ for $30 \mathrm{~s}$. The efficiency of cDNA synthesis was estimated using hGAPDH as a house-keeping gene. All data were analyzed via the comparative $C_{T}$ method [36], and all of the reactions were performed in triplicate.

\section{Cell proliferation assays}

REH-empty vector or REH-FAK shRNA cells $\left(4 \times 10^{5} /\right.$ $\mathrm{ml}$ ) were incubated with various concentrations of rapamycin for 48 or $72 \mathrm{~h}$, respectively, in 96-well plates (Costor, USA). After culture, Cell Counting Kit-8 (CCK8, Dojindo Molecular Technologies, Shanghai, China) solution $(10 \mu \mathrm{l})$ was added to each well, followed by incubation at $37^{\circ} \mathrm{C}$ for an additional $2 \mathrm{~h}$. The absorbance was measured at 450 and $630 \mathrm{~nm}$ using an absorbance reader. All experiments were performed in triplicate and were repeated at least three times.

\section{Cell apoptosis and cell cycle analysis}

Apoptosis was assessed through annexin $\mathrm{V} /$ propidium iodide (PI) staining. After transduced REH cells were treated with or without rapamycin $(100 \mathrm{nM})$ for $30 \mathrm{~h}$, they were stained with annexin V/PI (BD Biosciences, CA, USA) following the manufacturer's instructions and then analyzed via flow cytometry. Cell cycle analysis was performed on transduced REH cells incubated with or without rapamycin $(100 \mathrm{nM})$ for $48 \mathrm{~h}$. The cells were subsequently collected and incubated with $0.5 \mathrm{ml}$ of NP40/PI buffer and RNase $(25 \mu \mathrm{g} / \mathrm{ml})$ for $30 \mathrm{~min}$ at $37{ }^{\circ} \mathrm{C}$. Analysis by flow cytometry was performed immediately thereafter.

\section{In vivo experiments}

Male NOD/SCID mice were purchased from the Huafukang Company (Beijing, China) and were maintained in the animal center of Sun Yat-sen University under specific pathogen-free conditions. The murine model of leukemia was established as previously described [19]. Briefly, five million REH-FAK shRNA or REH-empty vector cells were injected into 6- to 8week-old NOD/SCID mice via the tail vein. For the in vivo assessment of drug effects, 10 days after transplantation of the REH cells, the mice were treated daily with $1.5 \mathrm{mg} / \mathrm{kg}$ rapamycin (or DMSO for the control group) via intraperitoneal injection for 7 days. Survival was monitored daily. Full blood counts were performed once a week. Several mice were sacrificed 25 days after transplantation, and their spleens were removed. The animal study was approved by the ethics committees of the animal center of Sun Yat-sen University.

\section{Statistical analysis}

The results are expressed as the mean \pm standard deviation (S.D.). The differences between groups were analyzed using Student's $t$ test when only two groups were compared or one-way analysis of variance (ANOVA) when more than two groups were compared. Log-rank $p$ values were determined using the Kaplan-Meier method comparing survival curves. Values of $p \leq 0.05$ were considered statistically significant.

\section{Abbreviations \\ ALL: acute lymphoblastic leukemia; AML: acute myeloid leukemia; BAK: BCL-2 antagonist killer; BAX: BCL-2-associated X protein; BCL-2: B-cell CLL/ lymphoma-2; BCR/ABL: breakpoint cluster region/Abelson leukemia virus; BIK: BCL-2 interacting killer; BMF: BCL-2-modifying factor; CCK-8: Cell Counting Kit-8; DMSO: dimethyl sulfoxide; ECL: enhanced chemiluminescence; FAK: focal adhesion kinase; GFP: green fluorescent protein; HSCT: hematopoietic stem cell transplantation; MCL-1: myeloid cell leukemia-1; mTOR: mammalian target of rapamycin; NOD/SCID: non-obese diabetic/severe combined immunodeficiency; PCR: polymerase chain reaction; PI: propidium iodide; PI3K: phosphatidylinositol 3-kinase; PUMA: p53 up-regulated modulator of apoptosis; PVDF: polyvinylidene fluoride; RIPA: radio immuno-precipitation assay; SDS: sodium dodecyl sulfate; shRNA: short-hairpin RNA; TBST: Tris-buffered saline Tween-20; WBC: white blood cell.}

\section{Competing interests \\ The authors declare that they have no competing interests.}

\section{Authors' contributions}

PS designed the study and carried out the cellular experiments and drafted the manuscript. LX participated in the design of the study and performed the in vivo experiments and helped to draft the manuscript. $\mathrm{KL}$ carried out the molecular experiments and helped with the in vitro and in vivo experiments. WW collected the clinical samples and helped with the statistical analysis. JF conceived of the study and participated in its design and coordination and helped to draft the manuscript. All authors read and approved the final manuscript.

\section{Acknowledgements}

This work was supported by the National Natural Science Foundation of China $(81100370,81570140)$

The authors wish to thank Professor Chen Yueqin and Professor Su Peiqiang who provided us with a laboratory to carry out the experiments. We thank technician Wang Ying at the Second Affiliated Hospital of Sun Yat-sen University, who offered us the machine for full blood cell counting. We thank technician Wu Shouhai at the Department of Life Science of Sun Yat-sen University, who helped us operate the flow cytometer. We thank the volunteers of the Second Affiliated Hospital of Sun Yat-sen 
University, who were willing to donate their bone marrow for research purposes. We thank Dr. Zeng Chenwu for giving us the primer for the BCL-2 family. We thank Dr. Gao Wenjie for helping us modify the manuscript. We thank Dr. Liao Yadi for helping us with the statistical analysis.

\section{Author details}

'Department of Pediatrics, Sun Yat-sen Memorial Hospital, Sun Yat-sen University, No. 107, West Yan Jiang Road, Guangzhou, Guangdong 510120, China. ${ }^{2}$ Department of Life Science, Sun Yat-sen University, No. 135, West Xin Gang Road, Guangzhou, Guangdong 510275, China.

\section{Received: 16 October 2015 Accepted: 9 February 2016 Published online: 18 February 2016}

\section{References}

1. Pui CH, Relling MV, Downing JR. Acute lymphoblastic leukemia. N Engl J Med. 2004;350(15):1535-48

2. Pui CH, Robison LL, Look AT. Acute lymphoblastic leukaemia. Lancet. 2008; 371(9617):1030-43.

3. Fan D, Li W, Yang Y, Zhang $X$, Zhang $Q$, Yan $Y$, et al. Redirection of CD4+ and CD8+ T lymphocytes via an anti-CD3 $\times$ anti-CD19 bi-specific antibody combined with cytosine arabinoside and the efficient lysis of patient-derived B-ALL cells. J Hematol Oncol. 2015:8(1):108.

4. Rai K. Therapeutic potential of new B cell-targeted agents in the treatment of elderly and unfit patients with chronic lymphocytic leukemia. J Hematol Oncol. 2015;8(1):85.

5. Wu J, Fu J, Zhang M, Liu D. Blinatumomab: a bispecific T cell engager (BiTE) antibody against CD19/CD3 for refractory acute lymphoid leukemia. J Hematol Oncol. 2015;8(1):104

6. Krentz S, Hof J, Mendioroz A, Vaggopoulou R, Dorge P, Lottaz C, et al. Prognostic value of genetic alterations in children with first bone marrow relapse of childhood B-cell precursor acute lymphoblastic leukemia. Leukemia. 2013;27(2):295-304

7. Daly A, Savoie ML, Geddes M, Chaudhry A, Stewart D, Duggan P, et al. Fludarabine, busulfan, antithymocyte globulin, and total body irradiation for pretransplantation conditioning in acute lymphoblastic leukemia: excellent outcomes in all but older patients with comorbidities. Biol Blood Marrow Transplant. 2012;18(12):1921-6.

8. Bassan R, Rossi G, Pogliani EM, Di Bona E, Angelucci E, Cavattoni I, et al. Chemotherapy-phased imatinib pulses improve long-term outcome of adult patients with Philadelphia chromosome-positive acute lymphoblastic leukemia: Northern Italy Leukemia Group protocol 09/00. J Clin Oncol. 2010; 28(22):3644-52.

9. Mohty M, Labopin M, Volin L, Gratwohl A, Socie G, Esteve J, et al. Reduced-intensity versus conventional myeloablative conditioning allogeneic stem cell transplantation for patients with acute lymphoblastic leukemia: a retrospective study from the European Group for Blood and Marrow Transplantation. Blood. 2010;116(22):4439-43.

10. Dantal J, Soulillou JP. Immunosuppressive drugs and the risk of cancer after organ transplantation. N Engl J Med. 2005;352(13):1371-3.

11. Guba M, von Breitenbuch P, Steinbauer M, Koehl G, Flegel S, Hornung $M$, et al. Rapamycin inhibits primary and metastatic tumor growth by antiangiogenesis: involvement of vascular endothelial growth factor. Nat Med. 2002:8(2):128-35.

12. Dumont FJ, Staruch MJ, Koprak SL, Melino MR, Sigal NH. Distinct mechanisms of suppression of murine $T$ cell activation by the related macrolides FK-506 and rapamycin. J Immunol. 1990;144(1):251-8.

13. Koehl GE, Andrassy J, Guba M, Richter S, Kroemer A, Scherer MN, et al. Rapamycin protects allografts from rejection while simultaneously attacking tumors in immunosuppressed mice. Transplantation. 2004; 77(9):1319-26.

14. Barrett D, Brown VI, Grupp SA, Teachey DT. Targeting the PI3K/AKT/mTOR signaling axis in children with hematologic malignancies. Paediatr Drugs. 2012;14(5):299-316.

15. Cabodi S, Di Stefano P, Leal MP, Tinnirello A, Bisaro B, Morello V, et al. Integrins and signal transduction. Adv Exp Med Biol. 2010;674:43-54.

16. Cox BD, Natarajan M, Stettner MR, Gladson CL. New concepts regarding focal adhesion kinase promotion of cell migration and proliferation. J Cell Biochem. 2006;99(1):35-52.
17. Schwock J, Dhani N, Hedley DW. Targeting focal adhesion kinase signaling in tumor growth and metastasis. Expert Opin Ther Targets. 2010;14(1):77-94

18. Tavernier-Tardy E, Cornillon J, Campos L, Flandrin P, Duval A, Nadal N, et al. Prognostic value of CXCR4 and FAK expression in acute myelogenous leukemia. Leuk Res. 2009;33(6):764-8.

19. Le $Y, X u$ L, Lu J, Fang J, Nardi V, Chai L, et al. FAK silencing inhibits leukemogenesis in BCR/ABL-transformed hematopoietic cells. Am J Hematol. 2009:84(5):273-8.

20. Fu QF, Liu Y, Fan Y, Hua SN, Qu HY, Dong SW, et al. Alpha-enolase promotes cell glycolysis, growth, migration, and invasion in non-small cell lung cancer through FAK-mediated PI3KAKT pathway. J Hematol Oncol. 2015;8(1):22

21. Sonoda Y, Watanabe S, Matsumoto Y, Aizu-Yokota E, Kasahara T. FAK is the upstream signal protein of the phosphatidylinositol 3-kinase-Akt survival pathway in hydrogen peroxide-induced apoptosis of a human glioblastoma cell line. J Biol Chem. 1999;274(15):10566-70.

22. Liu Z, Zhang HM, Yuan J, Lim T, Sall A, Taylor GA, et al. Focal adhesion kinase mediates the interferon-gamma-inducible GTPase-induced phosphatidylinositol 3-kinase/Akt survival pathway and further initiates a positive feedback loop of NF-kappaB activation. Cell Microbiol. 2008; 10(9):1787-800.

23. Glodek AM, Le Y, Dykxhoorn DM, Park SY, Mostoslavsky G, Mulligan R, et al. Focal adhesion kinase is required for CXCL12-induced chemotactic and proadhesive responses in hematopoietic precursor cells. Leukemia. 2007;21(8): 1723-32.

24. He X, Wang Y, Zhu J, Orloff M, Eng C. Resveratrol enhances the anti-tumor activity of the mTOR inhibitor rapamycin in multiple breast cancer cell lines mainly by suppressing rapamycin-induced AKT signaling. Cancer Lett. 2011; 301(2):168-76

25. Gangadhar TC, Cohen EE, Wu K, Janisch L, Geary D, Kocherginsky M, et al. Two drug interaction studies of sirolimus in combination with sorafenib or sunitinib in patients with advanced malignancies. Clin Cancer Res. 2011;17(7):1956-63.

26. Gomez-Martin C, Bustamante J, Castroagudin JF, Salcedo M, Garralda E, Testillano $\mathrm{M}$, et al. Efficacy and safety of sorafenib in combination with mammalian target of rapamycin inhibitors for recurrent hepatocellular carcinoma after liver transplantation. Liver Transpl. 2012;18(1):45-52.

27. Schwarzlose-Schwarck S, Scholz CW, Regierer AC, Martus P, Neumann C, Habbel $P$, et al. The mTOR inhibitor everolimus in combination with carboplatin in metastatic breast cancer - a phase I trial. Anticancer Res. 2012:32(8):3435-41.

28. Recher C, Beyne-Rauzy O, Demur C, Chicanne G, Dos SC, Mas VM, et al. Antileukemic activity of rapamycin in acute myeloid leukemia. Blood. 2005; 105(6):2527-34

29. Ikezoe T, Nishioka C, Bandobashi K, Yang Y, Kuwayama Y, Adachi Y, et al. Longitudinal inhibition of PI3K/Akt/mTOR signaling by LY294002 and rapamycin induces growth arrest of adult T-cell leukemia cells. Leuk Res. 2007;31(5):673-82.

30. Pan N, Zhang AR, Mu MS, Hou YC. Effects of FAK expression level on proliferation and motility of colorectal carcinoma cells. Xi Bao Yu Fen Zi Mian Yi Xue Za Zhi. 2009;25(9):783-6.

31. Cang S, Iragavarapu C, Savooji J, Song Y, Liu D. ABT-199 (venetoclax) and BCL-2 inhibitors in clinical development. J Hematol Oncol. 2015; 8(1):129.

32. François RA, Maeng K, Nawab A, Kaye FJ, Hochwald SN, Zajac-Kaye M Targeting focal adhesion kinase and resistance to mTOR inhibition in pancreatic neuroendocrine tumors. J Natl Cancer Inst. 2015;107(8):1-11. djv123.

33. Webber PJ, Park C, Qui M, Ramalingam SS, Khuri FR, Fu H, et al. Combination of heat shock protein 90 and focal adhesion kinase inhibitors synergistically inhibits the growth of non-small cell lung cancer cells. Oncoscience. 2015;2(9):765-76.

34. Tiscornia G, Singer O, Verma IM. Design and cloning of lentiviral vectors expressing small interfering RNAs. Nat Protoc. 2006;1(1):234-40.

35. Tiscornia G, Singer O, Verma IM. Production and purification of lentiviral vectors. Nat Protoc. 2006;1(1):241-5

36. Livak KJ, Schmittgen TD. Analysis of relative gene expression data using real-time quantitative PCR and the 2(-Delta Delta C(T)) Method. Methods. 2001;25(4):402-8. 